\title{
Implicit one-dimensional discrete maps and their connection with existence problem of chaotic dynamics in 3-D systems of differential equations
}

\author{
Vasiliy Ye. Belozyorov \\ Department of Applied Mathematics, Dnepropetrovsk National University, Gagarin's avenue, 72, 49050 Dnepropetrovsk, Ukraine
}

\section{A R T I C L E I N F O}

\section{Keywords:}

Implicit one-dimensional discrete map

Ordinary autonomous differential equations

system

Limit cycle

Chaotic attractor

\begin{abstract}
A B S T R A C T
New types of chaotic attractors for some 3-D autonomous systems of ordinary quadratic differential equations are founded. Examples are given.
\end{abstract}

(c) 2012 Elsevier Inc. All rights reserved.

\section{Introduction}

Chaos, as a very interesting complex nonlinear phenomenon, has been intensively studied in the last four decades in the science, mathematics and engineering communities. Recently, the chaos has been found to be very useful and having a great potential in many technological disciplines, such as information and computer sciences, power systems protection, biomedical systems analysis, flow dynamics and liquid mixing, encryption and communications, and so on. It is not surprising, therefore, that academic researches on chaotic dynamics has evolved from traditional trends of analyzing and understanding chaos to new directions of controlling and utilizing it (see, for example Refs. [1-13] and many references cited therein).

However there are only a few publications in which (from the mathematical point of view) the existence of chaotic dynamics is rigorously proved. It is known that a numerical evidence may occasionally be misleading, since computer simulations have finite precision and experimental measurements have finite ranges in the time or frequency domain. The witnessed behavior may be an artifact of the observation device due to physical limitations. Thus, a rigorous proof is often necessary for full understanding of the chaotic dynamics in various nonlinear dynamic systems.

The present work is a continuation of the article [2]. Its appearance is dictated by the desire to generalize results derived in [2] and, simultaneously, to do these results more rigorous.

The general theory of $n$-dimensional implicit discrete mappings was represented in [14]. It was based on a study of the special explicit positive and negative mappings.

In the present paper for the analysis of 1-dimensional implicit discrete mappings, generated by the Ricker discrete population model [2], other approach is offered. A contingency proof between the Ricker mapping [2] and some 1-dimensional explicit discrete mapping with the known chaotic properties is basis of this approach.

We will consider the following system (see [2]):

$$
\left\{\begin{array}{l}
\dot{x}(t)=a_{1} x(t)+a_{11} y^{2}(t)+a_{12} y(t) z(t)+a_{22} z^{2}(t) \\
\dot{y}(t)=b_{1} y(t)+c_{1} z(t)+b x(t) y(t) \\
\dot{z}(t)=-c_{1} y(t)+b_{1} z(t)+c x(t) z(t)
\end{array}\right.
$$

E-mail address: belozvye@mail.ru 
By Disc $=a_{12}^{2}-4 a_{11} a_{22}$ denote the discriminant of the quadratic form $a_{11} y^{2}+a_{12} y z+a_{22} z^{2}$. For system (1) it is necessary to distinguish two cases: (1) Disc $>0$ and (2) Disc $\leqslant 0$.

(1) Let Disc $>0$. In this case we have $a_{11} y^{2}+a_{12} y z+a_{22} z^{2}=(\alpha y+\beta z)(\gamma y+\delta z)$, where $\alpha, \beta, \gamma, \delta$ are real known constants. Introduce new variables $u$ and $v$ defined by the formulas $u=\alpha y+\beta z, v=\gamma y+\delta z$. Assume that $\Delta=\alpha \delta-\beta \gamma \neq 0$. Then in these variables system (1) will take the form

$$
\left\{\begin{array}{l}
\dot{x}(t)=a_{1} x(t)+u(t) v(t) \\
\dot{u}(t)=r_{1} u(t)+p_{1} v(t)+x(t)\left(r_{2} u(t)+p_{2} v(t)\right) \\
\dot{v}(t)=q_{1} u(t)+h_{1} v(t)+x(t)\left(q_{2} u(t)+h_{2} v(t)\right) .
\end{array}\right.
$$

where $r_{1}=\left(b_{1} \delta-c_{1} \gamma\right) / \Delta, \quad p_{1}=\left(-b_{1} \beta+c_{1} \alpha\right) / \Delta, \quad r_{2}=b \delta / \Delta, \quad p_{2}=-b \beta / \Delta, \quad q_{1}=\left(-c_{1} \delta-b_{1} \gamma\right) / \Delta, \quad h_{1}=\left(c_{1} \beta+b_{1} \alpha\right) / \Delta, \quad q_{2}=-c \gamma / \Delta$, $h_{2}=c \alpha / \Delta$.

Suppose that in system (2) $r_{2}=h_{2}=0$. (It can be if $\alpha=\delta=0$ ). Then we obtain a family of the systems similar to families which were investigated previously in numerous works (see, for example [15-21]).

For example in [16] the system

$$
\left\{\begin{array}{l}
\dot{x}(t)=a x(t)-y(t) z(t) \\
\dot{y}(t)=-b y(t)+x(t) z(t) \\
\dot{z}(t)=m x(t)-c z(t)+x(t) y(t)
\end{array}\right.
$$

was considered. In this three-dimensional autonomous system with simple system structure a new chaotic $2 \times 2$-scroll attractor was generated.

In [17-19] the system

$$
\left\{\begin{array}{l}
\dot{x}(t)=-(a b /(a+b)) x(t)-y(t) z(t)-c \\
\dot{y}(t)=a y(t)+x(t) z(t) \\
\dot{z}(t)=b z(t)+x(t) y(t)
\end{array}\right.
$$

was analyzed. For system (4) the existence of two homoclinic orbits was proved. It was shown that system (4) has a chaotic attractor of homoclinic type, when parameters of the system satisfy some conditions.

At last in [4] has proposed the new 3-D autonomous quadratic system

$$
\left\{\begin{array}{l}
\dot{x}(t)=a(y(t)-x(t))+e y(t) z(t) \\
\dot{y}(t)=c x(t)+d y(t)-x(t) z(t) \\
\dot{z}(t)=-b z(t)+x(t) y(t)
\end{array}\right.
$$

which can generate a four-wing chaotic attractor with complicated topological structures.

Thus, systems (3)-(5) are special cases of system (1).

(2) Now let Disc $\leqslant 0$. As far as we know this case was first considered in [2]. We mark that results got in [2] are incomplete. Therefore this case is the theme of further researches and in the present work it is not considered.

Further it will be shown that with the exception of known attractors, which were found in [2], system (1) possesses chaotic attractors are not indicated in [2]. The existence of these attractors is explained by a presence in the dynamic of system (1) an implicit iterated process of the Ricker type [2].

\section{Research of implicit function $F(x, y)=y \cdot \exp (\alpha \cdot y)-\lambda \cdot x \cdot \exp (\beta \cdot x)=0$}

(A) From the beginning in this section a few basic concepts of one-dimensional maps will be introduced.

By definition, put $\mathbb{V}=[0, \infty)$. Introduce on the space $\mathbb{V}$ a metric $d$ by the rule: $\forall v_{1}, v_{2} \in \mathbb{V} d=\left\|v_{1}-v_{2}\right\|$. Define by $h: \mathbb{V} \rightarrow \mathbb{V}$ an explicit function under the formula $h(v)=v \cdot \exp (r-v)$, where $r>0, v \in \mathbb{V}$.

Let $v^{*} \neq 0$ be a fixed point of $h$. It is obvious that $v^{*}=r$.

Denote by $B_{\delta}(a)=\{v \in \mathbb{V}: d(v, a)<\delta\}$ and $\overline{B_{\delta}}(a)=\{v \in \mathbb{V}: d(v, a) \leqslant \delta\}$ open and closed balls in $\mathbb{V}$. It is clear that $a \geqslant \delta \geqslant 0$.

Definition 1 [12]. A point $v^{*} \in \mathbb{V}$ is called an expanding fixed point of $h$ in $\overline{B_{\delta}}\left(v^{*}\right)$ for some constant $\delta>0$, if $h\left(v^{*}\right)=v^{*}$ and there exists a constant $\lambda>1$ such that

$$
d(h(x), h(y)) \geqslant \lambda d(x, y), \quad \forall x, y \in \overline{B_{\delta}}\left(v^{*}\right) .
$$

Furthermore, $v^{*}$ is called a regular expanding fixed point of $h$ in $\overline{B_{\delta}}\left(v^{*}\right)$ if $v^{*}$ is an interior point of $h\left(B_{\delta}\left(v^{*}\right)\right)$. 
Definition 2 [12] Assume that $v^{*}$ is an expanding fixed point of $h$ in $\overline{B_{\delta}}\left(v^{*}\right)$ for some $\delta>0$. Then, $v^{*}$ is said to be a snap-back repeller of $h$ if there exists a point $v_{0} \in B_{\delta}\left(v^{*}\right)$ with $v_{0} \neq v^{*}$ and $h^{(m)}\left(v_{0}\right)=v^{*}$ for some positive integer $m$. Furthermore, $v^{*}$ is said to be a non-degenerate snap-back repeller of $h$ if there exist positive constants $\mu$ and $\delta_{0}$ such that $B_{\delta_{0}}\left(v_{0}\right) \subset B_{\delta}\left(v^{*}\right)$ and

$$
d\left(h^{(m)}(x), h^{(m)}(y)\right) \geqslant \mu d(x, y), \quad \forall x, y \in \overline{B_{\delta_{0}}}\left(v_{0}\right) .
$$

Definition 3 [12]. The point $v^{*}$ is called a regular snap-back repeller of $h$ if $h\left(B_{\delta}\left(v^{*}\right)\right)$ is open and there exists a positive constant $\gamma_{0}$ such that $B_{\gamma_{0}}\left(v_{0}\right) \subset B_{\delta}\left(v^{*}\right)$ and $v^{*}$ is an interior point of $h^{(m)}\left(B_{\gamma}\left(v_{0}\right)\right)$ for any positive $\gamma<\gamma_{0}$.

Theorem 1 12. Assume that $h: \mathbb{V} \rightarrow \mathbb{V}$ has a regular and non-degenerate snap-back repeller $v^{*}$, associated with $v_{0} ; m$ and $\delta$ as specified in Definitions $1-3, h$ is continuous in $\overline{B_{\delta}}\left(v^{*}\right), h^{(m)}(v)$ is continuous in a neighborhood of $v_{0}$. Further, $h$ is continuous in some neighborhoods of $v_{1}, \ldots, v_{m-1}$, where $v_{j}=f^{j}\left(v_{0}\right)$ for $j=1, \ldots, m-1$. Then, there exists a compact and perfect invariant set $D \subset \mathbb{V}$ containing a Cantor set such that $h$ is chaotic in the sense of Devaney on $D$ as well as in the sense of Li-Yorke, and it has a dense orbit in $D$.

Now for the function $h(v)$ we find parameters which are indicated in Definitions 1-3. According to Theorem 1 it will prove that the map $h$ is chaotic.

(a1) Estimate a distance between points $h\left(v^{*}-\delta\right)$ and $h\left(v^{*}+\delta\right)$, where $\delta>0$. We have

$$
\begin{aligned}
d\left(h\left(v^{*}-\delta\right), h\left(v^{*}+\delta\right)\right)= & \left\|\left(v^{*}-\delta\right) \exp \left(r-\left(v^{*}-\delta\right)\right)-\left(v^{*}+\delta\right) \exp \left(r-\left(v^{*}+\delta\right)\right)\right\| \\
= & \| v^{*} \exp \left(r-v^{*}\right) \cdot \exp \delta-\delta \cdot \exp \left(r-v^{*}\right) \exp \delta-v^{*} \cdot \exp \left(r-v^{*}\right) \cdot \exp (-\delta) \\
& -\delta \cdot v^{*} \exp \left(r-\left(v^{*}+\delta\right)\right) \| .
\end{aligned}
$$

Since $v^{*} \exp \left(r-v^{*}\right)=v^{*}$ from the last formula it follows that

$$
\begin{aligned}
d\left(h\left(v^{*}-\delta\right), h\left(v^{*}+\delta\right)\right) & =\| r \cdot(\exp (\delta)-\exp (-\delta))-\delta \cdot(\exp (\delta)+\exp (-\delta) \| \geqslant 2 \delta \cdot(r-0.5 \cdot(\exp (\delta)+\exp (-\delta))) \\
& =\lambda \cdot d\left(v^{*}-\delta, v^{*}+\delta\right),
\end{aligned}
$$

where $\lambda=r-0.5 \cdot(\exp (\delta)+\exp (-\delta))$.

Let $\exp (\delta)=u$. Assume that $r-0.5 \cdot(\exp (\delta)+\exp (-\delta))=\lambda>1$. From here it follows that $u^{2}-2(r-1) u+1<0$. This inequality has the solution $1 \leqslant u<[r-1+\sqrt{r(r-2)}]$. Thus, if $0 \leqslant \delta<\ln [r-1+\sqrt{r(r-2)}]$ and $r>2$, then $\lambda>1$.

(a2) We have $h^{\prime}(v)=(1-v) \cdot \exp (r-v)$. A maximum point of the function $h(v)$ may be found from the condition $h^{\prime}(v)=0$. From here it follows that $v_{\max }=1$ and $h_{\max }=\exp (r-1)$. Thus, if $r>1$ then the function $h(v)$ will be non-monotone and unimodal on this interval: the interval $[0,1)$ is an increasing interval and the interval $(1, \infty)$ is a decreasing interval. Taking into account item (a1) we get that at $r>2$ the point $v^{*}=r$ is a repeller $\left(\left\|h^{\prime}(r)\right\|>1\right.$ at $\left.r>2\right)$. It is clear that $v^{*}$ is a regular expanding fixed point of $h$ in $\overline{B_{\delta}}\left(v^{*}\right)$.

(a3) Consider the process

$$
v_{i+1}=v_{i} \exp \left(r-v_{i}\right) ; \quad i=0,1,2, \ldots,
$$

where $r>0, v_{i}>0 ; i=0,1,2, \ldots$

By definition, put $\mathbb{W}=[0,1]$. Let $\mathbf{T}: \mathbb{V} \rightarrow \mathbb{W}$ be a continuous map given by the formula $w=(2 / \pi) \cdot \arctan v$. Since $\lim _{v \rightarrow \infty}(2 / \pi) \cdot \arctan v=1$ then we can consider that $\mathbf{T}$ is a homeomorphism and $\mathbf{T}(\mathbb{V})=\mathbb{W}, \mathbf{T}^{-1}(\mathbb{W})=\mathbb{V}$. By

$$
g(w) \equiv \mathbf{T}^{-1}\left(h(\mathbf{T}(w))=\frac{2}{\pi} \arctan \left(\tan \frac{\pi w}{2} \cdot \exp \left(r-\tan \frac{\pi w}{2}\right)\right)\right.
$$

define the continuous conjugate to $h$ mapping $g: \mathbb{W} \rightarrow \mathbb{W}[13]$.

The function $g(w)$ is similar to the function $h(v): w_{\max }=0.5$ and $g_{\max }=(2 / \pi) \cdot \arctan \exp (r-1)<1\left(\lim _{r \rightarrow \infty} g_{\max }=1\right)$; the interval $[0,0.5)$ is an increasing interval and the interval $(0.5,1]$ is a decreasing interval. Therefore, if $w^{*}=r>0.5$ then $(2 /$ $\pi) \cdot \arctan \exp (r-1)>(2 / \pi) \cdot \arctan r$. From here it follows that $\exp (r-1)>r$ and $r>1$.

It is clear that the inverse mapping $g^{-1}(w)$ has two branches: $g_{1}^{-1}(w)$ and $g_{2}^{-1}(w)$, where each of the mappings $g_{1}^{-1}(w)$ and $g_{2}^{-1}(w)$ is invertible. Define the function $G: \mathbb{W} \rightarrow \mathbb{W}$ by the rule

$$
G(w)=g_{i_{1}}^{-1}\left(g_{i_{2}}^{-1}\left(\ldots\left(g_{i_{k}}^{-1}(w)\right)\right)\right), \quad k=2,3, \ldots
$$

where either $i_{k}=1$ or $i_{k}=2$. It is clear that the mapping $G(w)$ is monotonically.

The mapping $G(w)$ has at least one fixed point $w^{*}$. It is known that the fixed point $w^{*}$ of the mapping $G(w)$ corresponds to a fixed point $w^{* *}$ of the mapping $g^{(k)}(w)=g(g(\ldots g(w)))$. The point $w^{* *}$ of the mapping $g^{(k)}(w)$ corresponds to either a fixed point or a $p$-cycle of the mapping $g(w)$. If $w^{* *}$ is a fixed point of $g(w)$ then it is possible only at $i_{1}=i_{2}$. Since it is possible to take any integer $k$ and arbitrarily choose numbers $i_{1}, i_{2}$ from 1 to 2 , then from the condition $g_{1}^{-1}\left(g_{2}^{-1}(w)\right) \neq g_{2}^{-1}\left(g_{1}^{-1}(w)\right)$ it follows that the non-monotone function $g(w)$ can have any number of cycles of different multiples and an uncountable set of non-periodic trajectories. 


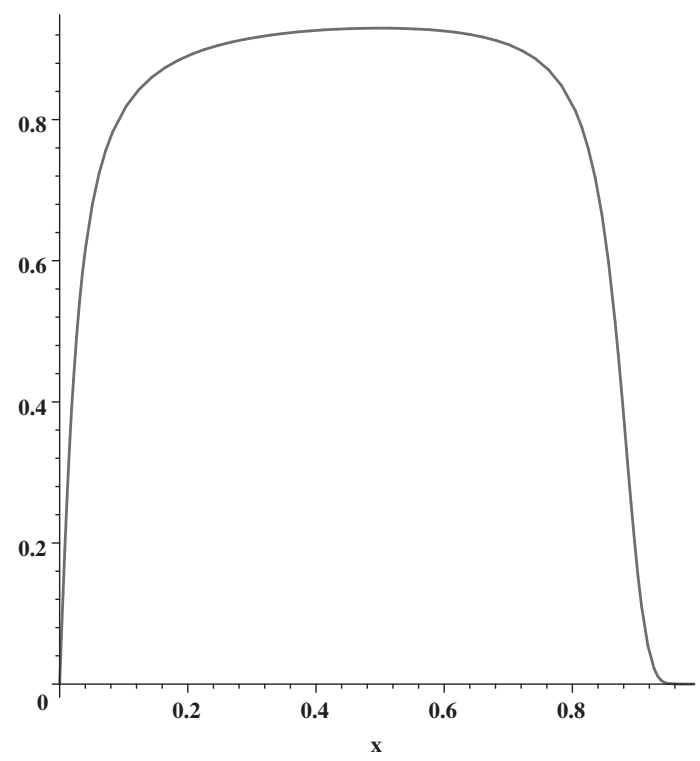

Fig. 1. The mapping $g(x) ; r=3.2$.

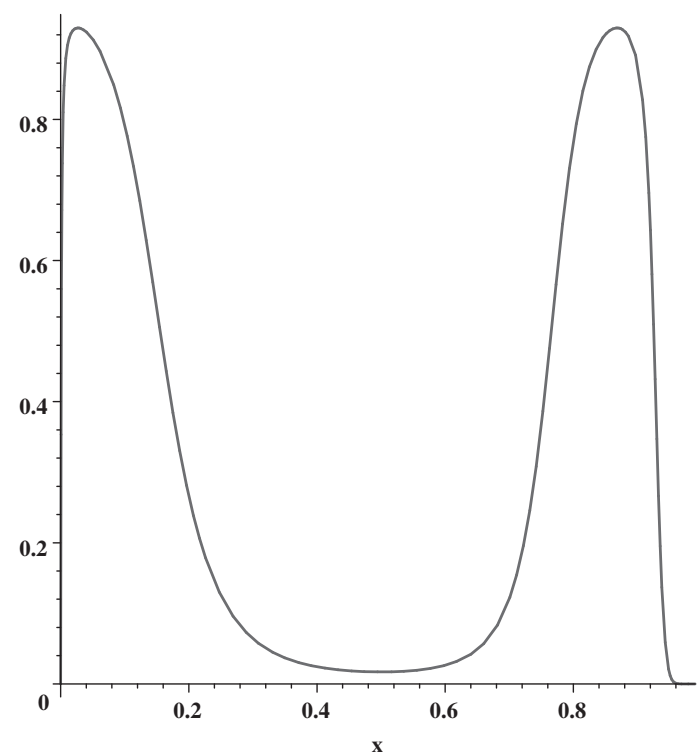

Fig. 2. The mapping $g(g(x)) ; r=3.2$.

We show that at some values of the parameter $r$ function (7) can be chaotic. For this purpose we specify those values of the parameter $r$ at which in system $w_{i+1}=g\left(w_{i}\right), i=0,1,2, \ldots$, a 3-cycle appears.

The equations $w=g(w), w=g(g(w))$, and $w=g(g(g(w)))$ are being solved difficult. Therefore, we use by the conjugation of $g$ and $h$. (On Figs. 1-4 two iterations of mappings $g$ and $h$ are shown.)

Let $v=h(v) \equiv v \exp (r-v)$. Then at $r>1$ in system (6) there exists 1 -cycle. Let $v=h(h(v)) \equiv v \exp (2 r-v-v \exp (r-v))$. In this case at $r>2.008$ in system (6) there exist 1-cycles and 2-cycles. Now let $v=h(h(h(v))) \equiv v \exp (2 r-v-v \exp (r-v)) \cdot \exp$ $(r-v \exp (r-v) \exp (r-v \exp (r-v)))$. In this case at $r>3.103$ in system (6) there exist 1-cycles, 2-cycles, and 3-cycles. (The indicated values $r$ can be found by any mathematical package which has a numeral solution program of transcendental equations.)

According to Sharkovsky's Theorem [13] a cycle of period 3 implies cycles of all periods. Thus, in system $w_{i+1}=g\left(w_{i}\right)$ there exist all cycles with period $2^{i}, i=0,1,2, \ldots$ According to Singer's Theorem [13] in the discrete system $w_{i+1}=g\left(w_{i}\right)$, $i=0,1,2, \ldots$, at any $n$ and some value of parameter $r=r_{n}$ there are all unstable cycles of period $2^{i}, i=0, \ldots, n-1$, and one 


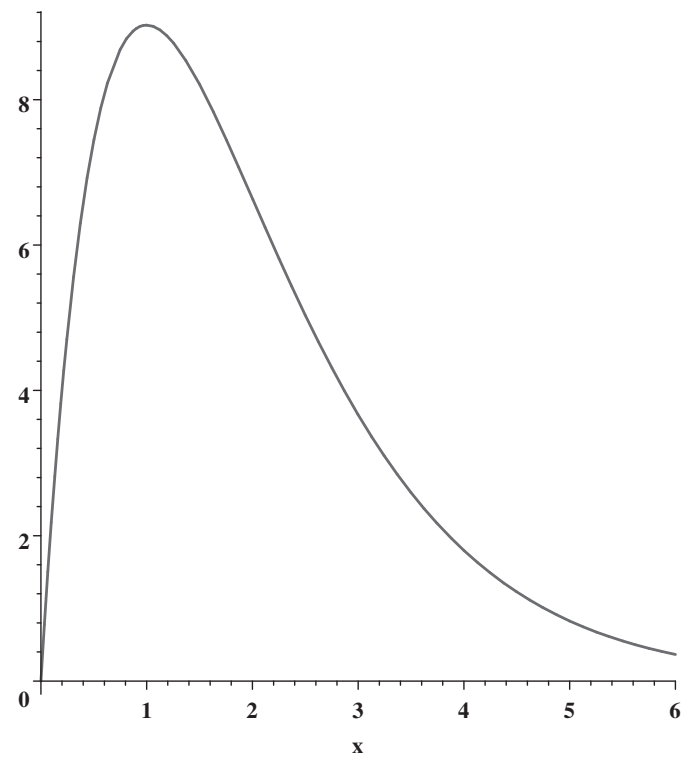

Fig. 3. The mapping $h(x) ; r=3.2$.

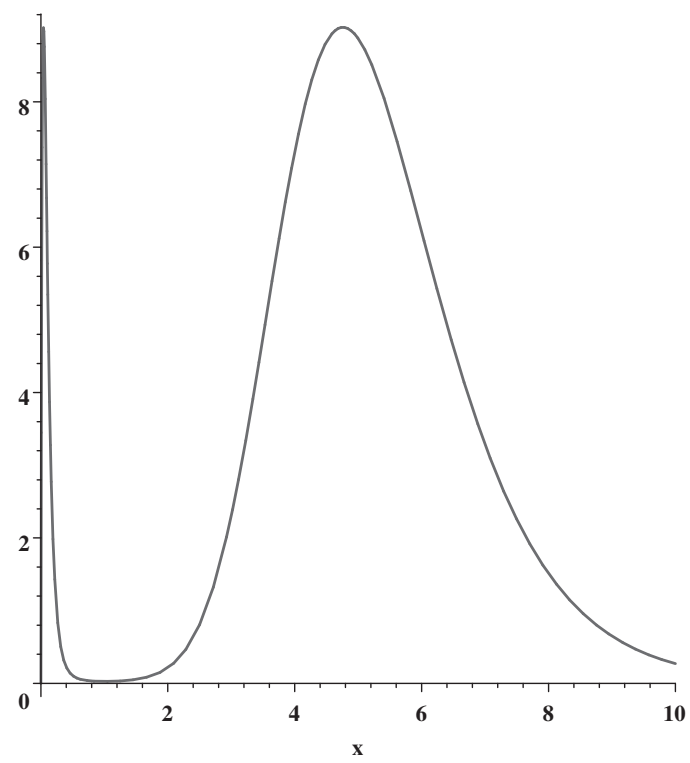

Fig. 4. The mapping $h(h(x)) ; r=3.2$.

stable cycle of period $2^{n}$. If $r=r_{\infty}=3.103$ then mapping $g(w)$ has a semi-stable trajectory $\mathbb{S}$ in any neighborhood of any point of this trajectory lie points of a countable set of unstable cycles of all periods $2^{i}, i=0,1,2, \ldots$. Therefore, a set of all periodic points is density in $\mathbb{W}$. (Any point of $\mathbb{S}$ with given accuracy can be approximated by some periodic point.)

(a4) In this item Definitions 1-3 will be carried on the space $\mathbb{W}$ and the map $g: \mathbb{W} \rightarrow \mathbb{W}$. Let $w^{*} \neq 0$ be a fixed point of $g$. Assume that $r>3.103$. Then for any the iterated function $g^{(m)}(w)$ on interval $[0,1]$ there are $2^{m}$ fixed points $w_{0}^{*}=0, w_{1}^{*}, \ldots, w_{l-1}^{*}$, where $l=2^{m}$. Among these points there is the fixed point $w^{*}$.

Suppose that $w_{l-2}^{*}=w^{*}$. It is obvious that the equation $g^{(m)}(w)=w^{*}$ has also $2^{m}$ real roots $\xi_{0}, \ldots \xi_{l-1}$ on interval $[0,1]$. The space $\mathbb{W}$ is bounded. Therefore, the distance between two nearest fixed points tends to $0: \lim _{m \rightarrow \infty} d\left(w^{*}, w_{l-1}^{*}\right)=0$. It means that $\lim _{m \rightarrow \infty} d\left(w^{*}, \xi_{l-1}\right)=0$.

It is clear that $g\left(\xi_{l-2}\right)=g\left(w^{*}\right)$. From here it follows that there exists a point $w_{0}$ such that $\xi_{l-2}=g^{(m-1)}\left(w_{0}\right)$ and $g\left(g^{(m-1)}\left(w_{0}\right)=g\left(g^{(m-1)}\left(w^{*}\right)=w^{*}\right.\right.$. Furthermore, we have $\lim _{m \rightarrow \infty} d\left(w^{*}, w_{0}\right)=0$. 
(a5) Let us remind that by virtue of contingency of the maps $g$ and $h$ all assertions which are valid for $g$ take place and for $h$ [13].

With the help of the operator $\mathbf{T}^{-1}$ we return from the space $\mathbb{W}$ to the space $\mathbb{V}$. Then there exists the point $v_{0}=\mathbf{T}^{-1}\left(w_{0}\right)$ for which the conditions of Definition 2 are fulfilled.

Indeed, let $v^{*}=h^{(m)}\left(v_{0}\right)$ and

$$
\overline{B_{\delta_{2}}}\left(v_{0}\right) \subset \overline{B_{\delta_{1}}}\left(v^{*}\right) \subset \overline{B_{\delta}}\left(v^{*}\right), \delta_{2}<\delta_{1}<\delta,
$$

be a sequence of embedded balls. We have

$$
d\left(h^{(m)}(x), h^{(m)}(y)\right) \geqslant \lambda d\left(h^{(m-1)}(x), h^{(m-1)}(y)\right) \geqslant \ldots \geqslant \lambda^{m} d(x, y), \forall x, y \in \overline{B_{\delta_{1}}}\left(v^{*}\right) .
$$

It is clear that $\mu=\lambda^{m}$ and $\delta_{2}^{m}<\delta_{1}^{m}<\delta$. Consider the sequence $\left\{v_{j}=h^{(j)}\right\}, j=0, \ldots, m$, where $v_{j} \in \overline{B_{\delta_{1}}}\left(v^{*}\right)$. Let $\delta_{2}=\max$ $\left(d\left(v_{0}, v_{1}\right), \ldots, d\left(v_{0}, v_{m}\right)\right)$. According to item (a 4$)$ for large enough $m$ the distance $d\left(v^{*}, v_{0}\right)$ may be arbitrarily small. Therefore,

$$
d\left(h^{(m)}(x), h^{(m)}(y)\right) \geqslant \mu d(x, y), \quad \forall x, y \in \overline{B_{\delta_{2}}}\left(v_{0}\right)
$$

and all conditions of Definition 2 are fulfilled. The point $v^{*}$ is the non-degenerate snap-back repeller of $h$.

Finally, we verify the realization of conditions of Definition 3. Suppose opposite: the point $v^{*}$ is not an interior point of $h^{(m)}\left(B_{\gamma}\left(v_{0}\right)\right)$ for any positive $\gamma<\delta_{2}$. In this case the point $v^{*}$ belongs to boundaries of sets $\overline{h^{(m)}}\left(B_{\gamma}\left(v_{0}\right)\right), \overline{B_{\delta_{1}}}\left(v^{*}\right)$, and $\overline{B_{\delta}}\left(v^{*}\right)$. In this case we get $\delta=\ln [r-1+\sqrt{r(r-2)}], \lambda=1$, and $r=2$ in contradiction with supposition $r>2$. Thus, we have $\gamma_{0}=\delta_{2}$ and the point $v^{*}$ is a regular snap-back repeller of $h$. It means that all conditions of Theorem 1 is satisfied. Therefore, the map $h: \mathbb{V} \rightarrow \mathbb{V}$ is chaotic.

(B) Further, in this section we will consider that variables $x \geqslant 0, y \geqslant 0$, and parameters $\lambda>0, \alpha \in \mathbb{R}, \beta \in \mathbb{R}$.

The following theorem can be found in any textbook on advanced calculus or mathematical analysis.

Theorem 2 (Implicit function Theorem). Assume that a function $H(x, y): \mathbb{R} \times \mathbb{R} \rightarrow \mathbb{R}$ is continuously differentiable at each point $(x, y)$ of an open set $\mathbb{D} \subset \mathbb{R} \times \mathbb{R}$. Let $\left(x_{0}, y_{0}\right)$ be a point in $\mathbb{D}$ for which $H\left(x_{0}, y_{0}\right)=0$ and for which $H_{y}^{\prime}\left(x_{0}, y_{0}\right) \neq 0$. Then there exist neighborhoods $U \subset \mathbb{R}$ of $x_{0}$ and $V \subset \mathbb{R}$ of $y_{0}$ such that for each $x \in U$ the equation $H(x, y)=0$ has a unique solution $y \in V$. Moreover, this solution can be given as $y=h(x)$, where $h(x)$ is continuously differentiable at $x=x_{0}$.

Let us define singular points of the function $F(x, y)=y \cdot \exp (\alpha \cdot y)-\lambda \cdot x \cdot \exp (\beta \cdot x)=0$. These points are roots of the system equations $F_{x}^{\prime}(x, y)=0, F_{y}^{\prime}(x, y)=0$. We get a unique singular point $\left(x_{s}, y_{s}\right)=(-1 / \beta,-1 / \alpha)$. Since $x \geqslant 0, y \geqslant 0$, then at $\beta>0$, $\alpha>0$, at $\beta>0, \alpha<0$, and at $\beta<0, \alpha>0$ the first quadrant does not contain of singular points.

Let $\beta<0, \alpha<0$. We calculate a discriminant Disc of the function $F(x, y)=y \cdot \exp (\alpha \cdot y)-\lambda \cdot x \cdot \exp (\beta \cdot x)=0$ at $(x, y)=\left(x_{s}, y_{s}\right)$ :

$$
\text { Disc }=F_{x x}^{\prime \prime} \cdot F_{y y}^{\prime \prime}-\left(F_{x y}^{\prime \prime}\right)^{2}=-\lambda \alpha \beta \cdot \exp (\alpha x+\beta y)<0 .
$$

Thus, if $\beta<0, \alpha<0$, then the function $F(x, y)=0$ can have a self-intersection point. This point exists at implementation of the condition $F(-1 / \beta,-1 / \alpha)=\lambda /(\beta \cdot e)-1 /(\alpha \cdot e)=0$.

The last condition may be rewritten in the form $\beta-\alpha \lambda=0$.

Consider the following cases: (a) $\beta-\alpha \lambda<0, \alpha<0, \beta<0$; (b) $\beta-\alpha \lambda>0, \alpha<0, \beta<0$; (c) $\alpha>0, \beta<0$; (d) $\alpha<0, \beta>0$.

We have

$$
y_{x}^{\prime}=-\frac{F_{x}^{\prime}}{F_{y}^{\prime}}=\lambda \frac{1+\beta \cdot x}{1+\alpha \cdot y} \exp (\beta \cdot x-\alpha \cdot y) .
$$

(a) Let $y \cdot \exp (\alpha \cdot y)=\lambda \cdot x \cdot \exp (\beta \cdot x)=c \geqslant 0$. Then the equation $\lambda \cdot x \cdot \exp (\beta \cdot x)=c$ has two roots. Therefore, the function $F(x, y)=y \cdot \exp (\alpha \cdot y)-\lambda \cdot x \cdot \exp (\beta \cdot x)=0$ has two branches.

Let $1+\beta \cdot x=1+\alpha \cdot y=0$. It is means that $x=x_{s}=-1 / \beta, y=y_{s}=-1 / \alpha$, and $F\left(x_{s}, y_{s}\right)=(e \alpha)^{-1}-\lambda(e \beta)^{-1}=0$. The last equation contradicts by the condition $\beta-\alpha \lambda<0$. Therefore, if $1+\beta \cdot x=0$, then $1+\alpha \cdot y \neq 0$.

If $\beta-\alpha \lambda=0$, then $y_{\max }=y_{\min }=-1 / \alpha$. Therefore, if $\beta-\alpha \lambda<0$, then $\forall x \in[0, \infty) y_{\max }<-1 / \alpha$ for the lower branch of the curve $F(x, y)=0$, and $y_{\min }>-1 / \alpha$ for the upper branch of the curve $F(x, y)=0$.

We have $y_{x}^{\prime}=0$ at $x=x_{s}=-1 / \beta$. As for $y \approx 0$ we have $1+\alpha \cdot y>0$, then by virtue of Implicit function Theorem the point $x=x_{s}=-1 / \beta$ is a maximum of the implicit function $y_{l}=f_{l}(x)$ (the lower branch) and a minimum of the implicit function $y_{u}=f_{u}(x)$ (the upper branch). The case (a) is shown on Fig. 5.

(b) This case repeats the case (a) if to replace $x$ by $y$. The point $y=y_{s}=-1 / \alpha$ is a maximum point for the left branch and a minimum point for the right branch of the curve $F(x, y)=0$. The case (b) is shown on Fig. 6.

(c) Let again $y \cdot \exp (\alpha \cdot y)=\lambda \cdot x \cdot \exp (\beta \cdot x)=c \geqslant 0$. In this case the equation $y \cdot \exp (\alpha \cdot y)=c$ has only one root. Therefore, the function $F(x, y)=y \cdot \exp (\alpha \cdot y)-\lambda \cdot x \cdot \exp (\beta \cdot x)=0$ has one branch (see Fig. 7).

(d) This case repeats the case (c) if to replace $x$ by $y$ (see Fig. 8). 


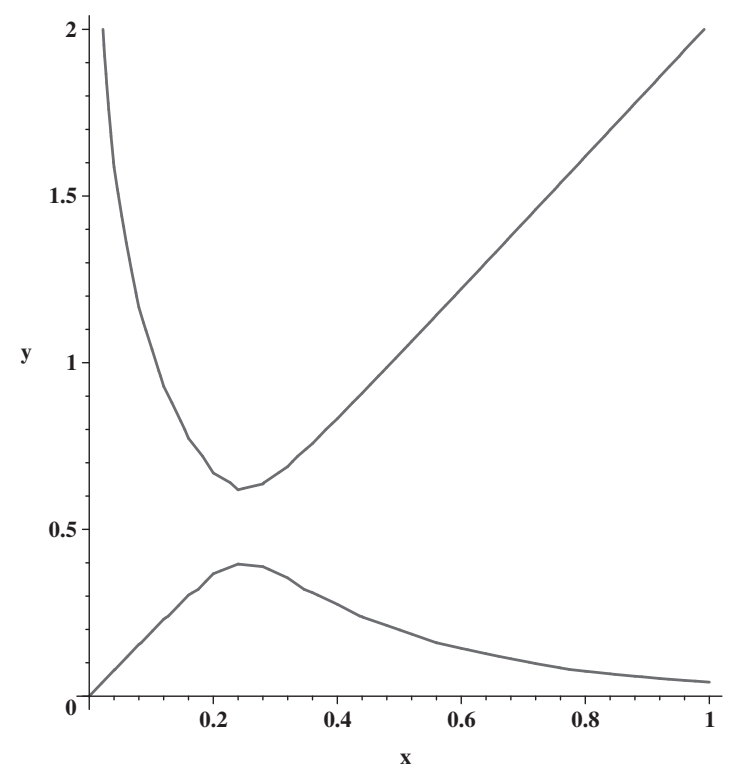

Fig. 5. The curve exp $y \cdot(-2 \cdot y)-1.95 \cdot x \cdot \exp (-4 \cdot x)=0$.

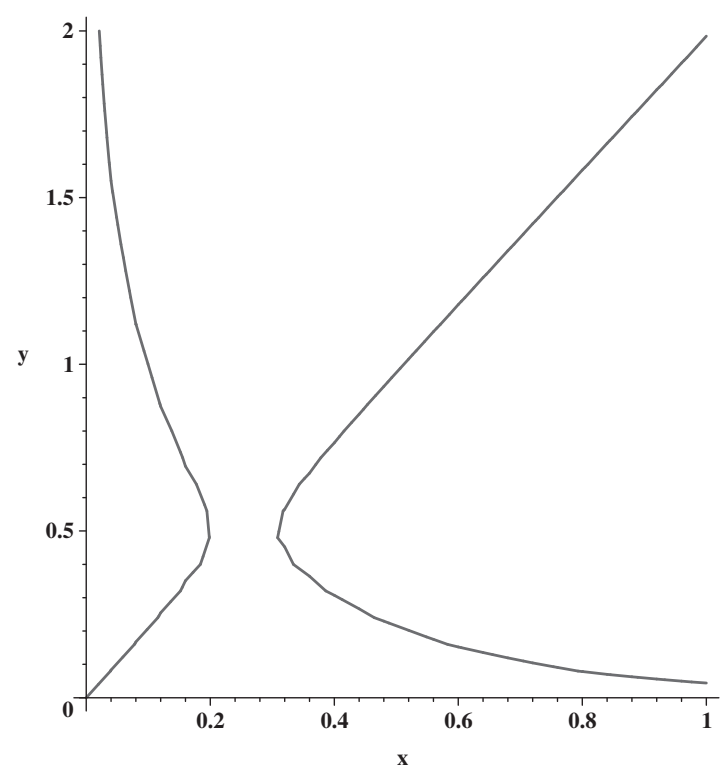

Fig. 6. The curve $y \cdot \exp (2 \cdot y)-2.05 \cdot x \cdot \exp (-4 \cdot x)=0$.

Notice that if either the condition (a) or the condition (c) is valid, then the magnitude $y$ is bounded $(y<-1 / \alpha)$. Since at $x \rightarrow \infty$ we have $y=\lambda \cdot x \cdot \exp (\beta \cdot x-\alpha \cdot y)<\lambda_{1} \cdot x \cdot \exp (\beta \cdot x)$, where $\lambda_{1}=\lambda \cdot e$, then the function $y=\lambda \cdot x \cdot \exp (\beta \cdot x-\alpha \cdot y)$ behaves also as the function $\lambda_{1} \cdot x \cdot \exp (\beta \cdot x)$ (here $\left.\beta<0\right)$.

In future, we will be interested by cases (a) and (c). Let $\beta=-p, \alpha=-m$, where $p>0$. Then the case (a) takes place at $-p+\lambda \cdot m<0$ and $0 \leqslant y<1 / m$; the case (c) takes place at $-p+\lambda \cdot m<0$ and $m<0$.

Let us rewrite the equation $F(x, y)=0$ in the form: $y=\lambda x \cdot \exp (-p x+m y)$.

Consider the implicit discrete mapping

$$
x_{n+1}=\lambda x_{n} \cdot \exp \left(-p x_{n}+m x_{n+1}\right), \quad n=0,1,2, \ldots
$$




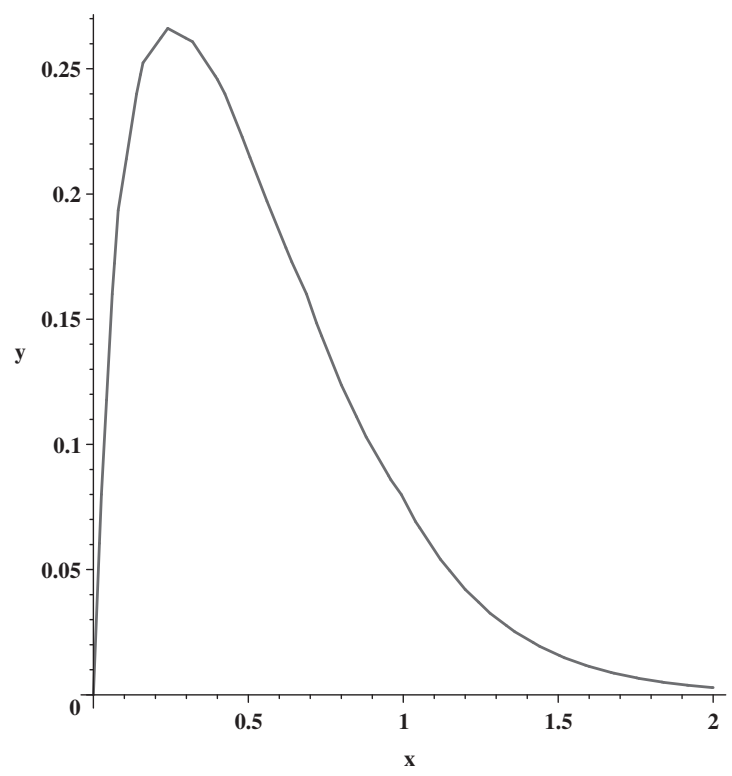

Fig. 7. The curve $y \cdot \exp (2 \cdot y)-5 \cdot x \cdot \exp (-4 \cdot x)=0$.

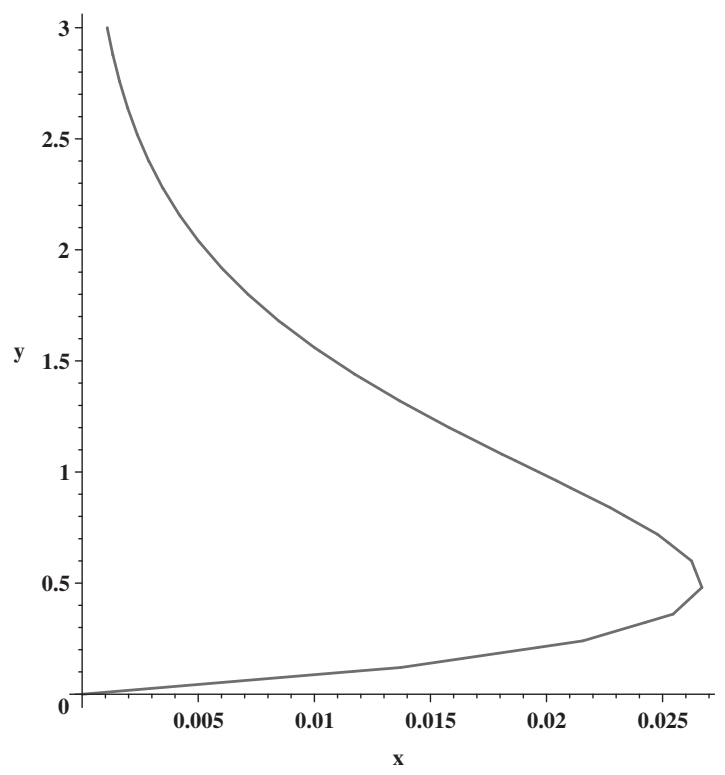

Fig. 8. The curve $y \cdot \exp (-2 \cdot y)-5 \cdot x \cdot \exp (4 \cdot x)=0$

Theorem 3. Assume that $p>0, \lambda>0, x_{0}>0$. Let either

(1) $m>0,-p+\lambda m<0$, and $x_{0}<1 / m$ (for example, $p=1, m=0.1$, and $\lambda_{c r}=9.025$ ) or

(2) $m \leqslant 0$ (for example, $p=1, m=0$, and $\lambda_{c r}=24.533 ; p=1, m=-0.1$, and $\lambda_{c r}=66.686$ ). Then mapping (8) is chaotic.

Proof. From conditions of Theorem 1 and Implicit function Theorem it follows that the explicit function $y=f(x)$ is derived from the implicit function $F(x, y)=0$ which satisfies by the condition: $f([0, \infty)) \subset[0, \infty)$.

Let in (8) $m \neq 0$. With the help of replacements $v_{i}=p x_{i}$ of variables $x_{i}$ the process (8) can be represented in the form

$$
v_{i+1}=v_{i} \exp \left(r-v_{i}+s v_{i+1}\right) ; \quad i=0,1,2, \ldots
$$

where $s=m / p, r=\ln \lambda>0, v_{i}>0 ; i=0,1,2, \ldots$. 


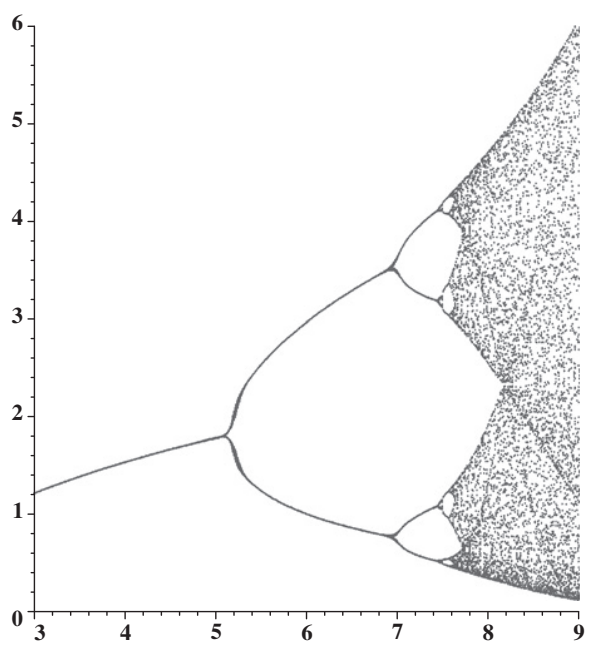

Fig. 9. The bifurcation diagram of the mapping $x_{n+1}=\lambda x_{n} \cdot \exp \left(-x_{n}+0.1 \cdot x_{n+1}\right) ; \lambda_{c r}=9.025$.

We fix the value $v_{0}=v_{0}^{*}$. Use Implicit function Theorem for deriving of solutions $v_{1}^{*}, v_{2}^{*}, \ldots$ of Eq. (9) with respect to $v_{i+1}$; $i=0,1,2, \ldots$. Then process (9) may be rewritten in the explicit form

$$
v_{i+1}=v_{i} \exp \left(r+s v_{i+1}^{*}-v_{i}\right) ; \quad i=0,1,2, \ldots
$$

Let $m>0(s>0)$. Then we have $v_{i}^{*}<1 / s$ and $r+s v_{i+1}^{*}<r+1$. Therefore, process (10) may be approximated by process

$$
t_{i+1}=t_{i} \exp \left(r+1-t_{i}\right) ; \quad i=0,1,2, \ldots
$$

Since the chaos arises up in system (11) at $r+1=3.103$, in system (10) it will arise up at $r-1=2.103$ (see items (a1) - (a5)). If $m<0(s<0)$ then process (6) is approximated by process (10). In this case $r-1=3.103<r-s v_{i+1}^{*}$ and the chaos arises up in system (10) at $r+1=4.103$. Taking into account that $\lambda=\ln r$, we get statements of Theorem 3. (On Figs. 9-11 bifurcation diagrams of the map $x_{n+1}=\lambda \cdot \exp \left(-x_{n}+s \cdot x_{n+1}\right)$ are shown.)

\section{Chaotic attractors of system (1)}

Consider the system

$$
\left\{\begin{array}{l}
\dot{x}(t)=a_{1} x(t)+a_{11} y^{2}(t)+a_{12} y(t) z(t)+a_{22} z^{2}(t), \\
\dot{y}(t)=d_{1} x(t)+b_{1} y(t)+c_{1} z(t)+b x(t) y(t), \\
\dot{z}(t)=e_{1} x(t)+f_{1} y(t)+g_{1} z(t)+c x(t) z(t),
\end{array}\right.
$$

where $a_{1}, \ldots, g_{1}, c \in \mathbb{R}$.

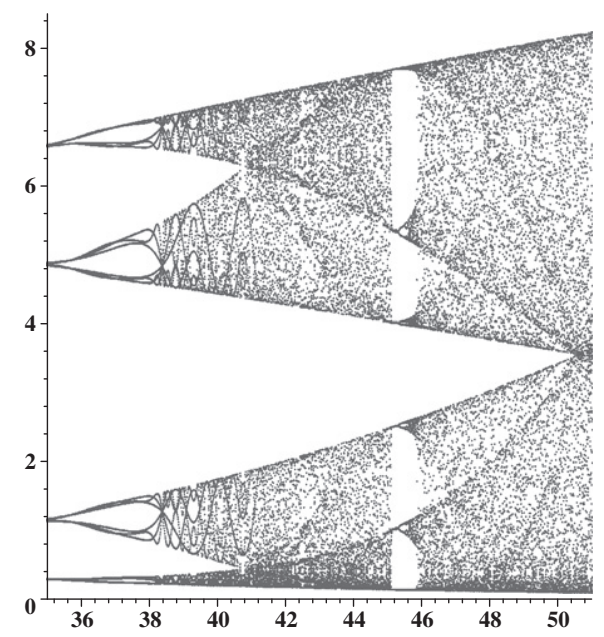

Fig. 10. The bifurcation diagram of the mapping $x_{n+1}=\lambda x_{n} \cdot \exp \left(-x_{n}-0.1 \cdot x_{n+1}\right) ; \lambda_{c r}=66.686$. 


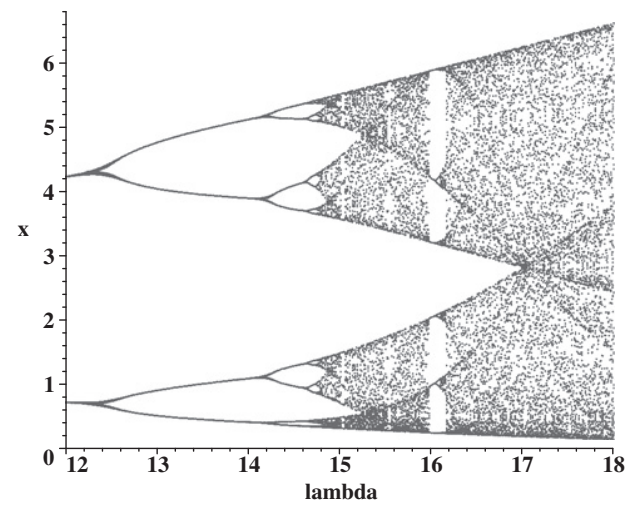

Fig. 11. The bifurcation diagram of the mapping $x_{n+1}=\lambda x_{n} \cdot \exp \left(-x_{n}\right) ; \lambda_{c r}=24.533$.

Theorem 4. Assume that for system (12) conditions $a_{11} / b<0, a_{22} / c<0$, and $a_{12}^{2}-a_{11} a_{22}>0$ are valid. Suppose also that either $b+c \neq 0, \frac{a_{12}^{2}}{(b+c)^{2}}-\frac{a_{11} a_{22}}{b c}<0$

or

$$
b+c=0, a_{12}=0 .
$$

Besides, we will consider that the matrix

$$
\left(\begin{array}{ccc}
0 & 0 & 0 \\
d_{1} & b_{1} & c_{1} \\
e_{1} & f_{1} & g_{1}
\end{array}\right)
$$

has the spectrum $\{0, \beta \pm i \gamma\}$, where $\beta<0, \gamma \neq 0, i=\sqrt{-1}$. Then there exists a set $W:=\left\{x_{0}, y_{0}, z_{0}\right\}$ of initial values such that $\forall\left(x_{0}, y_{0}, z_{0}\right) \in W$ the solution of system (12) with initial values $\left(x_{0}, y_{0}, z_{0}\right)$ is bounded.

Proof. 1.Introduce into system (1) the new variables $\rho$ and $\phi$ under the formulas: $y=\rho \cos \phi, z=\rho \sin \phi$, where $\rho>0$. Then, we get

$$
\left\{\begin{array}{l}
\dot{x}(t)=a_{1} \cdot x(t)+\rho^{2}(t) \cdot\left(a_{11} \cos ^{2} \phi(t)+a_{12} \cos \phi(t) \sin \phi(t)+a_{22} \sin ^{2} \phi(t)\right), \\
\dot{\rho}(t)=\left(b_{1}+\left(b \cos ^{2} \phi(t)+c \sin ^{2} \phi(t)\right) \cdot x(t)\right) \cdot \rho(t) \\
\dot{\phi}(t)=-c_{1}-(b-c) \cos \phi(t) \cdot \sin \phi(t) \cdot x(t)
\end{array}\right.
$$

where $a_{1}, a_{11}, a_{12}, a_{22}, b, c, b_{1}, c_{1} \in \mathbb{R}$.

Let $b_{1}=c_{1}=0$. Then system (14) takes the form

$$
\left\{\begin{array}{l}
\dot{x}(t)=a_{1} \cdot x(t)+\rho^{2}(t) \cdot\left(a_{11} \cos ^{2} \phi(t)+a_{12} \cos \phi(t) \sin \phi(t)+a_{22} \sin ^{2} \phi(t)\right), \\
\left.\dot{\rho}(t)=\left(b \cos ^{2} \phi(t)+c \sin ^{2} \phi(t)\right) \cdot x(t)\right) \cdot \rho(t), \\
\dot{\phi}(t)=-(b-c) \cos \phi(t) \cdot \sin \phi(t) \cdot x(t) .
\end{array}\right.
$$

From the second and third equations of system (15) it follows that

$$
\frac{d \rho}{\rho}=-\frac{1}{b-c} \frac{b \cos ^{2} \phi+c \sin ^{2} \phi}{\sin \phi \cos \phi} d \phi, \quad \ln \rho=-\frac{1}{b-c} \int \frac{b \cos ^{2} \phi+c \sin ^{2} \phi}{\sin \phi \cos \phi} d \phi,
$$

and

$$
\rho=\rho_{0}|\sin \phi|^{\frac{-b}{b-c}} \cdot|\cos \phi|^{\frac{c}{b-c}},
$$

where $\rho_{0}=\rho(0)>0, \frac{-b}{b-c}<0, \frac{c}{b-c}<0$. Then first equation of system (15) can be rewritten in the form 


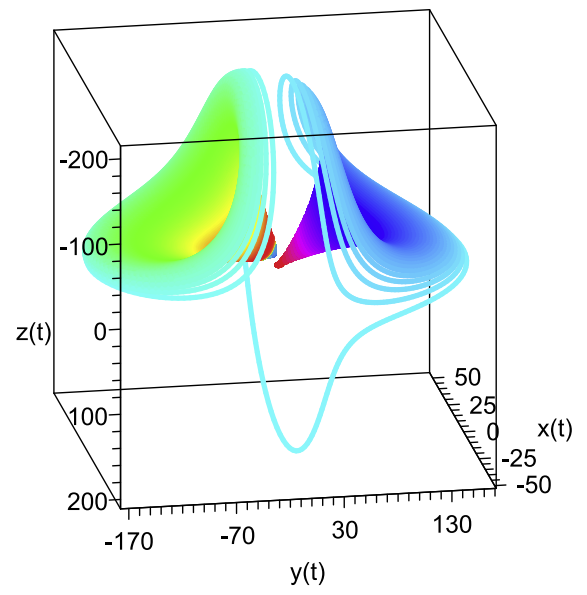

Fig. 12. The phase portrait of system (22) for $t=10$. Here $a=10, b=15, x_{0}=-0.01, y_{0}=-0.01, z_{0}=0.1$.

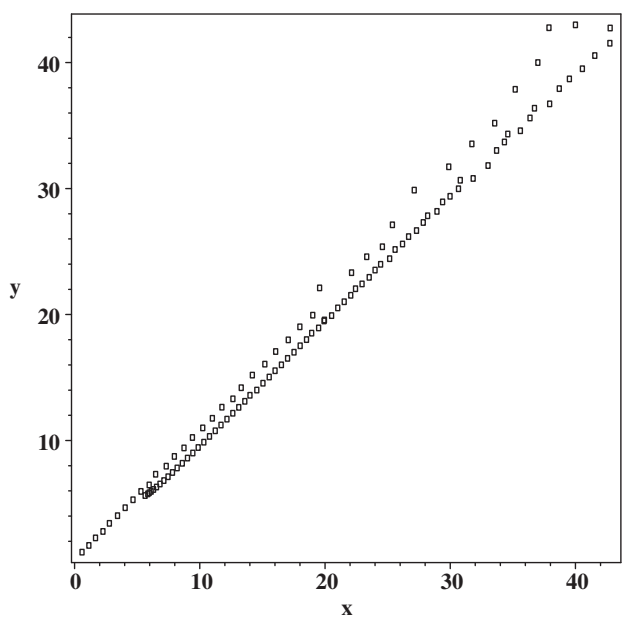

Fig. 13. The first-return map to a Poincare section for system (22) at $a=10, b=15$; here $\mathbf{x}=x_{n}, \mathbf{y}=x_{n+1}$ are sequential maximus of the function $x(t)$; $n \in\{1,2,3, \ldots\}$. The trajectory goes out on a limit cycle: $\lim _{n \rightarrow \infty} x_{n}=5.634$.

$$
\dot{x}(t)=a_{1} \cdot x(t)+\rho_{0}^{2}|\sin \phi(t)|^{\frac{-2 b}{b-c}} \cdot|\cos \phi(t)|^{\frac{2 c}{b-c}} \cdot\left(a_{11} \cos ^{2} \phi(t)+\cdots+a_{22} \sin ^{2} \phi(t)\right) .
$$

It is clear that all maximums $x_{\text {imax }}(i \rightarrow \infty)$ of the function $x(t)$ can be found from the condition $\dot{x}(t)=0$. By virtue of the condition $a_{12}^{2}-a_{11} a_{22}>0$ the equation $a_{11} \cos ^{2} \phi+a_{12} \sin \phi \cos \phi+a_{22} \sin ^{2} \phi=0$ has an infinite set of roots $\phi_{1}=\phi\left(t_{1}\right), \phi_{2}=\phi\left(t_{2}\right), \ldots$ Therefore, if $a_{1} \neq 0$, then $x_{i \max }$ satisfies by the condition

$$
x_{i \max } \cdot\left(\sin ^{2} \phi\left(t_{i}\right)\right)^{\frac{b}{b-c}} \cdot\left(\cos ^{2} \phi\left(t_{i}\right)\right)^{\frac{-c}{b-c}}=\frac{\rho_{0}^{2} \cdot\left(a_{11} \cos ^{2} \phi\left(t_{i}\right)+\cdots+a_{22} \sin ^{2} \phi\left(t_{i}\right)\right)}{-a_{1}} \equiv s_{i},
$$

where $\lim _{i \rightarrow \infty} t_{i}=\infty$. From here it follows that if $\lim _{i \rightarrow \infty} s_{i}=$ const $\neq 0$, then $\lim _{i \rightarrow \infty}\left|x_{i \max }\right|=\infty$ and $\lim i \rightarrow \infty \sin \phi\left(t_{i}\right) \cos \phi\left(t_{i}\right)=0$. If $\lim _{i \rightarrow \infty} s_{i}=0$, then $\lim _{i \rightarrow \infty}\left|x_{i \max }\right|=0$. At $a_{1}=0$ the boundedness of solutions for system (15) was proved in [2]. Thus, $\forall a_{1} \in \mathbb{R}$ there exist the initial values $\left(x_{0}, y_{0}, z_{0}\right)$ such that the solution of system (15) with these initial values is bounded.

2. We take advantage of item (c) from the proof of Theorem 4 [2]. Let us replace in this item the matrix

$$
\left(\begin{array}{ccc}
0 & 0 & 0 \\
0 & b_{1} & c_{1} \\
0 & -c_{1} & b_{1}
\end{array}\right)
$$

by matrix (13). 


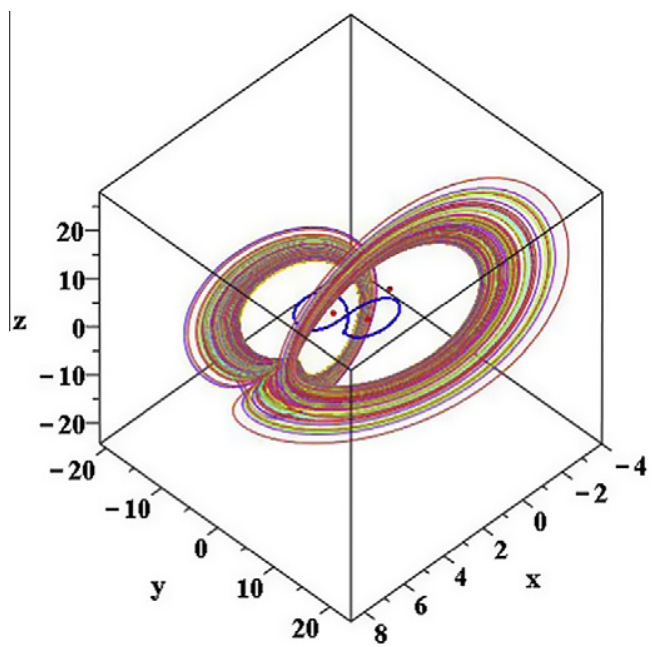

Fig. 14. The phase portrait of system (22) for $t=15$. Here $a=7, b=15$. There are two coexisting attractors; it is one chaotic attractor and one limit cycle.

Let us consider that for system (12) at $d_{1}^{2}+e_{1}^{2}=0$ the conditions of Theorem 4 are valid. Then Theorem 4 from this paper is an obvious generalization of Theorems 1,4 , and Theorem 6 [2]. (Notice that condition $a_{1}>0$ in the proofs of Theorem 4 and Theorem $6[2]$ is surplus.)

Now assume that $d_{1}^{2}+e_{1}^{2} \neq 0$. Then taking advantage of Gronwall-Bellman inequality by the same method as in the proof of Theorem 4 [2] it is possible to prove the boundedness of solutions of system (12) and at $d_{1}^{2}+e_{1}^{2} \neq 0$.

From the first equation of system (14) it follows that the equation $V(t) \equiv \dot{x}(t)-a_{1} x(t)=0$ has a countable number of roots $t_{1}, \ldots t_{i}, \ldots$, where $\phi\left(t_{i}\right)=k_{i} \pi ; k_{i}$ is an integer. It means that the function $V(t)$ changes a sign with period $\pi$. Hence, extremum points of function $x(t)$ (for example maximums) has a period $\phi\left(t_{i+2}\right)-\phi\left(t_{i}\right)=\pi$.

Let $x\left(t_{i}\right)=x_{i}, \rho\left(t_{i}\right)=\rho_{i}, \phi\left(t_{i}\right)=\phi_{i}$, where $t_{i}$ are roots of the first equation $\dot{x}\left(t_{i}\right)=0$ of system (14), $i=1,2, \ldots$ We can consider that $<\phi_{i-1}<\phi_{i}<\phi_{i+1}<\phi_{i+2}<\ldots$.

We will assume that one of the following two variants takes place:

either

(1) $t_{i}, t_{i+1}$, and $t_{i+2}$ are sequential a minimum, a maximum, and a minimum of the function $\rho(t) ; i=1,2, \ldots$ or

(2) $t_{i}, t_{i+1}$, and $t_{i+2}$ are sequential a maximum, a minimum, and a maximum of the function $x(t) ; i=1,2, \ldots$. Assume that the second variant takes place.

Theorem 5. Assume that $a_{1}>0$ and there exists a moment $t^{*}$ such that from the condition $t>t^{*}$ it follows that $x(t) \geqslant 0$. Let $\forall i \in\{1,3,5, \ldots\}$ the condition

$$
\left.\int_{t_{i}}^{t_{i+2}}\left[b+c+(b-c) \cos 2 \phi(\tau)-\frac{b_{1}(b-c)}{c_{1}} \sin 2 \phi(\tau)\right)\right] \cdot x(\tau) d \tau<0 .
$$

be fulfilled. Then under the conditions of Theorem 4 in system (1) there are either limit cycles or chaotic attractors.

Proof. From system (14) we have:

$$
x\left(t_{i}\right)=-\frac{\rho^{2}\left(t_{i}\right) \cdot\left(a_{11} \cos ^{2} \phi\left(t_{i}\right)+a_{12} \cos \phi\left(t_{i}\right) \sin \phi\left(t_{i}\right)+a_{22} \sin ^{2} \phi\left(t_{i}\right)\right)}{a_{1}},
$$

$i=1,3, \ldots$

Consider the fraction

$$
\frac{x_{i+2}}{x_{i}}=\frac{\rho_{i+2}^{2} \cdot\left(a_{11} \cos ^{2} \phi_{i+2}+a_{12} \cos \phi_{i+2} \sin \phi_{i+2}+a_{22} \sin ^{2} \phi_{i+2}\right)}{\rho_{i}^{2} \cdot\left(a_{11} \cos ^{2} \phi_{i}+a_{12} \cos \phi_{i} \sin \phi_{i}+a_{22} \sin ^{2} \phi_{i}\right)} .
$$

From (18) it follows that $\forall i$ the magnitude $\phi\left(t_{i+2}\right)-\phi\left(t_{i}\right)=\phi(T)$, where $\phi(T)$ is a period of the function $a_{11} \cos ^{2} \phi(t)+a_{12} \cos \phi(t)$ $\sin \phi(t)+a_{22} \sin ^{2} \phi(t)$. Then,

$$
\frac{a_{11} \cos ^{2} \phi_{i+2}+a_{12} \cos \phi_{i+2} \sin \phi_{i+2}+a_{22} \sin ^{2} \phi_{i+2}}{a_{11} \cos ^{2} \phi_{i}+a_{12} \cos \phi_{i} \sin \phi_{i}+a_{22} \sin ^{2} \phi_{i}}=1 .
$$




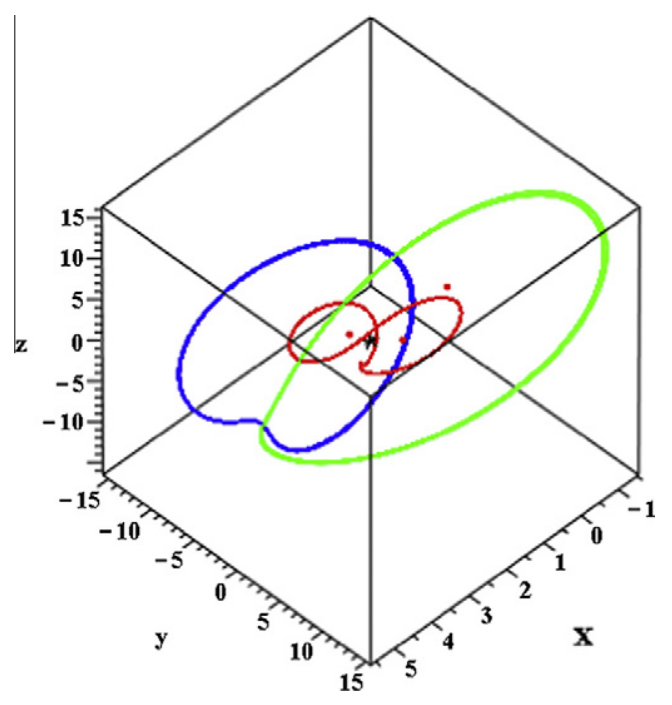

Fig. 15. The phase portrait of system (22) for $t=15$. Here $a=7.5, b=15$. There are three coexisting limit cycles.

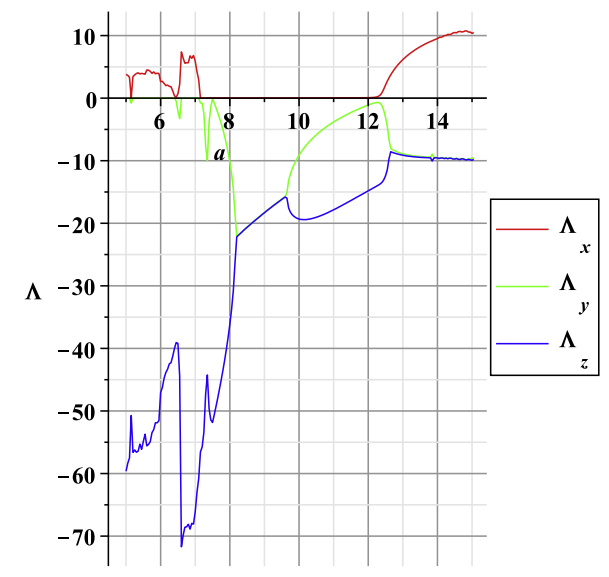

Fig. 16. Lyapunov exponents $\Lambda_{x}, \Lambda_{y}, \Lambda_{z}$ for system (22) at $a=5 \div 15, b=15$.

Any function of the kind $d_{11} \cos ^{2} \phi+d_{12} \cos \phi \sin \phi+d_{22} \sin ^{2} \phi$ always can be transformed to the form $g_{1}+g_{2} \cos 2 \phi+$ $g_{12} \sqrt{1-\cos ^{2} 2 \phi}$. Hence, the period of these functions is equal $\pi$. Then from the third equation of system (14), we have

$$
\phi\left(t_{i+2}\right)-\phi\left(t_{i}\right)=\pi=c_{1}\left(t_{i+2}-t_{i}\right)+\frac{b-c}{2} \int_{t_{i}}^{t_{i+2}} \sin 2 \phi(t) x(t) d t ; \quad c_{1}<0 .
$$

(If $c_{1}>0$, then $\phi\left(t_{i+2}\right)-\phi\left(t_{i}\right)=-\pi$.)

Therefore, the fraction $x_{i+2} / x_{i}$ may be rewritten as

$$
\begin{aligned}
\frac{x_{i+2}}{x_{i}} & \left.=\exp \left[\frac{2 b_{1} \pi}{c_{1}}+\int_{t_{i}}^{t_{i+2}}\left[b+c+(b-c) \cos 2 \phi(\tau)-\frac{b_{1}(b-c)}{c_{1}} \sin 2 \phi(\tau)\right)\right] \cdot x(\tau) d \tau\right] \\
& \left.=\lambda \exp \left[\int_{t_{i}}^{t_{i+2}}\left[b+c+(b-c) \cos 2 \phi(\tau)-\frac{b_{1}(b-c)}{c_{1}} \sin 2 \phi(\tau)\right)\right] \cdot x(\tau) d \tau\right],
\end{aligned}
$$

where $\lambda=\exp \left(2 b_{1} \pi / c_{1}\right)$.

Let us introduce the function

$$
h(\phi)=b+c+(b-c) \cos 2 \phi-\frac{b_{1}(b-c)}{c_{1}} \sin 2 \phi .
$$




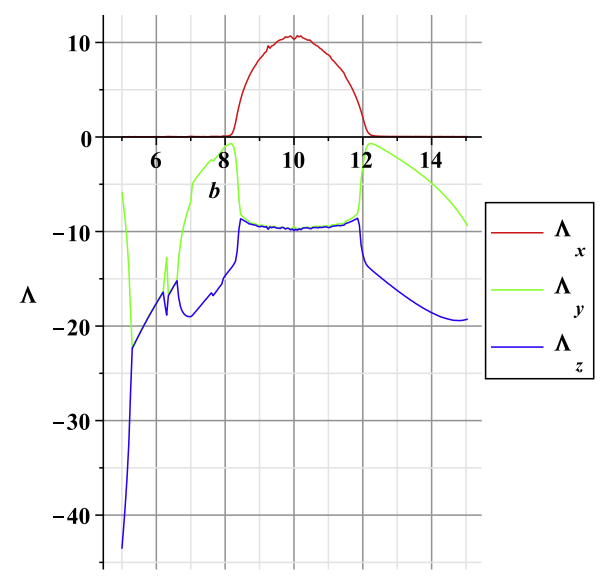

Fig. 17. Lyapunov exponents $\Lambda_{x}, \Lambda_{y}, \Lambda_{z}$ for system (22) at $a=10, b=5 \div 15$.

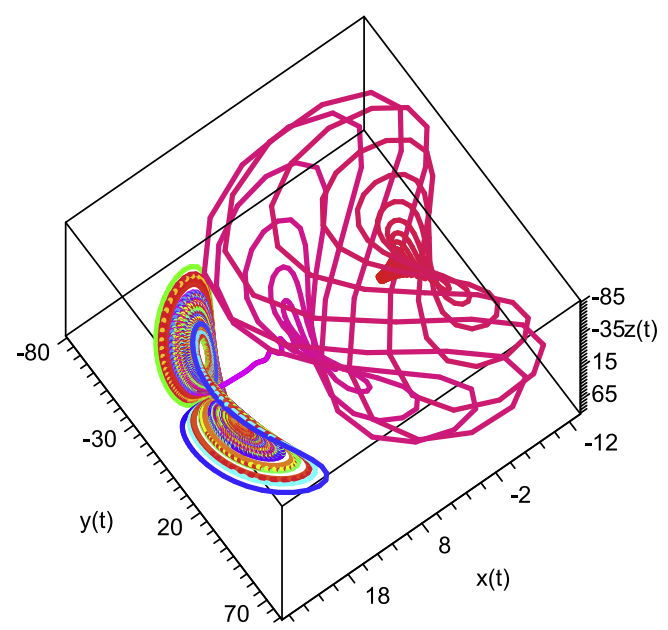

Fig. 18. The phase portrait of systems (23) for $t=10$. Here $c=10, d=15$. There is one chaotic attractor.

The bounded function $x(t)$ is a monotone decreasing on the interval $\left[t_{i}, t_{i+1}\right]$ and it is a monotone increasing on the interval $\left[t_{i+1}, t_{i+2}\right]$. Then we have (it us known Theorem about average value):

$$
\begin{aligned}
\int_{t_{i}}^{t_{i+2}} h(\phi(\tau)) \cdot x(\tau) d \tau & =\int_{t_{i}}^{t_{i+1}} h(\phi(\tau)) \cdot x(\tau) d \tau+\int_{t_{i+1}}^{t_{i+2}} h(\phi(\tau)) \cdot x(\tau) d \tau \\
& =x\left(t_{i}+0\right) \int_{t_{i}}^{\xi} h(\phi(\tau)) d \tau+x\left(t_{i+2}-0\right) \int_{\zeta}^{t_{i+2}} h(\phi(\tau)) d \tau,
\end{aligned}
$$

where $t_{i} \leqslant \xi_{i} \leqslant t_{i+1}, t_{i+1} \leqslant \zeta_{i+2} \leqslant t_{i+2}$. We can consider that $t^{*}<t_{i}$. Then the function $x(t)$ is positive on the interval $\left[t_{i}, t_{i+2}\right]$. Hence, from (19) it follows that

$$
\int_{t_{i}}^{t_{i+2}} h(\phi(\tau)) \cdot x(\tau) d \tau=p_{i} x_{i}+p_{i+2} x_{i+2}
$$

where the magnitudes $p_{i}=\int_{t_{i}}^{\xi_{i}} h(\phi(\tau)) d \tau, p_{i+2}=\int_{\xi_{i+2}}^{t_{i+2}} h(\phi(\tau)) d \tau$ can have any signs.

Finally, we get

$$
x_{i+2}=\lambda x_{i} \exp \left(p_{i} x_{i}+p_{i+2} x_{i+2}\right) ; \quad i=1,3,5, \ldots, 2 n-1, \ldots
$$

It is obvious that if $p_{i} x_{i}+p_{i+2} x_{i+2}<0$ then condition (17) is hold. Suppose that $p_{i}=-p<0, p_{i+2}=m \in \mathbb{R}$. By Theorem 4 all solutions of system (1) are bounded. Therefore, the conditions (a) and (c) of Section 2 can be united in one condition: $-p+\lambda m<0$ (see Section 2). For example, assume $\lambda=20, p_{i}=-4, p_{i+2}=-1$. Then after the first iteration, we have the equation $x=\lambda x \exp \left(\left(p_{i}+p_{i+2}\right) x\right)$; this equation has two roots: 0 , 0.6. After the second step, we get the equation 


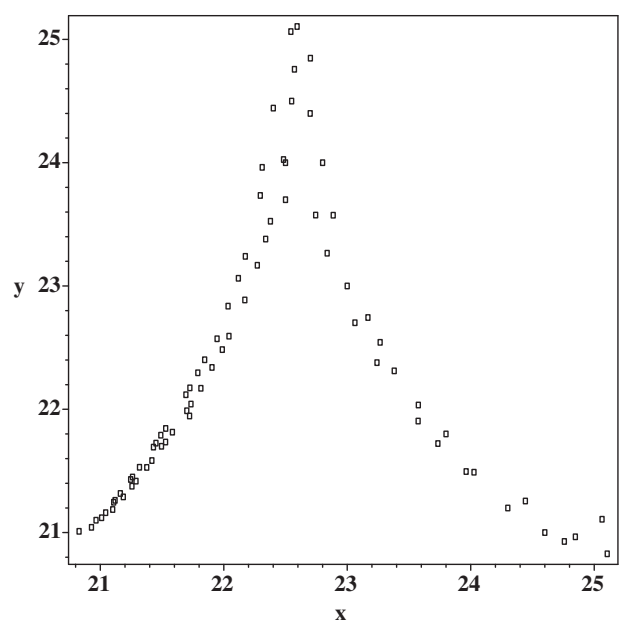

Fig. 19. The first-return map to a Poincare section for system (23) at $c=10, d=15$; here $\mathbf{x}=x_{n}, \mathbf{y}=x_{n+1}$ are sequential maximus of the function $x(t)$; $n \in\{1,2,3, \ldots\}$.

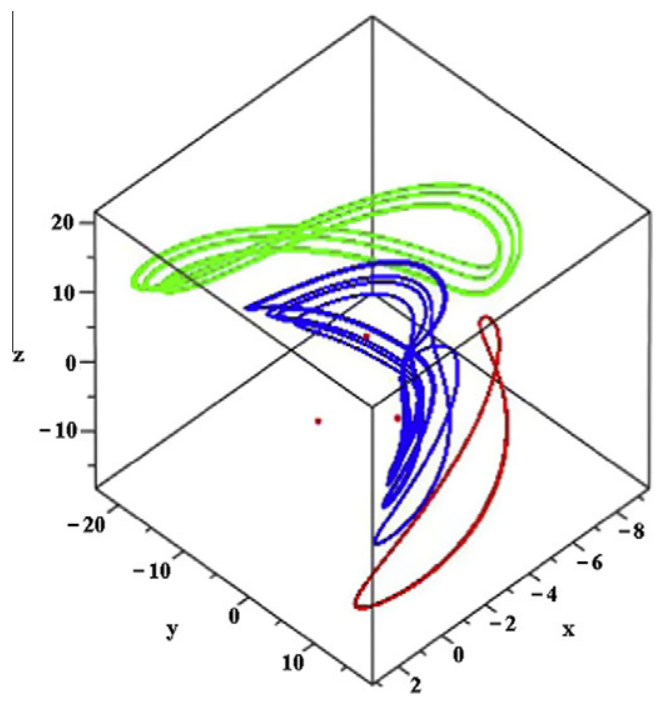

Fig. 20. The phase portrait of system (23) for $t=15$. Here $c=10, d=5$. There are three coexisting limit cycles.

$x=\lambda^{2} x \exp \left(\left(p_{i}+2 p_{i+2}\right) x+p_{i} \lambda \exp \left(\left(p_{i}+p_{i+2}\right) x\right)\right)$; this equation has four roots: $0,0.11,0.6,0.82$ and so on. Finally, we derive the Feigenbaum scenario of the doubling period. Since $a_{1}>0$ then equilibrium point $(0,0,0)$ is a saddle-focus. It means the existence in system (1) of limit cycles or chaotic attractors.

Theorem 5 can be generalized in the following way.

Assume that system (1) has a chaotic attractor or a limit cycle. If in system (12) the values $d_{1}=0, e_{1}=0$ are not bifurcations, then for small enough numbers $\left|d_{1}\right|$ and $\left|e_{1}\right|$ system (12) has also a chaotic attractor or a limit cycle.

Let $x_{i}=z_{i}>0, p_{i}=-1, p_{i+2}=0, x_{i+2}=z_{i+1}$. Then process (20) may be rewritten as:

$$
z_{i+1}=z_{i} \exp \left(r-z_{i}\right), r=\frac{2 \pi b_{1}}{c_{1}}>0, \quad i=0,1,2 \ldots
$$

Process (21) is called the Ricker discrete population model [22,23]. It is known [22] that the Ricker model has 2-cycle, 4-cycle and chaotic attractors when $r=2.1, r=2.6$, and $r=3$, respectively. 


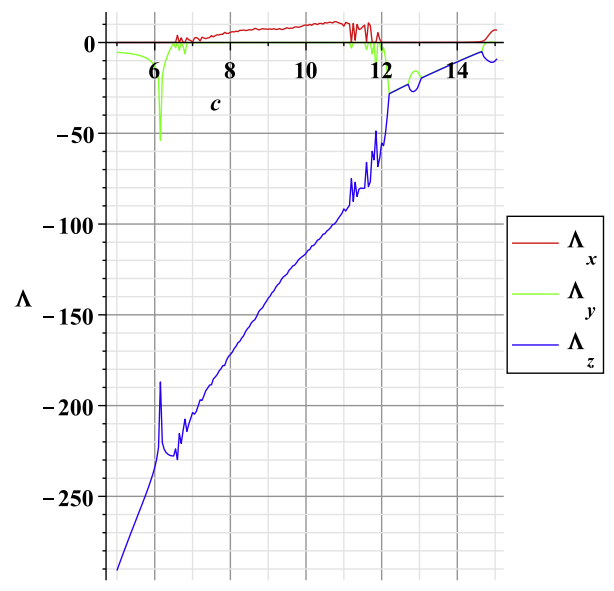

Fig. 21. Lyapunov exponents $\Lambda_{x}, \Lambda_{y}, \Lambda_{z}$ for system (23) at $c=5 \div 15, d=15$.

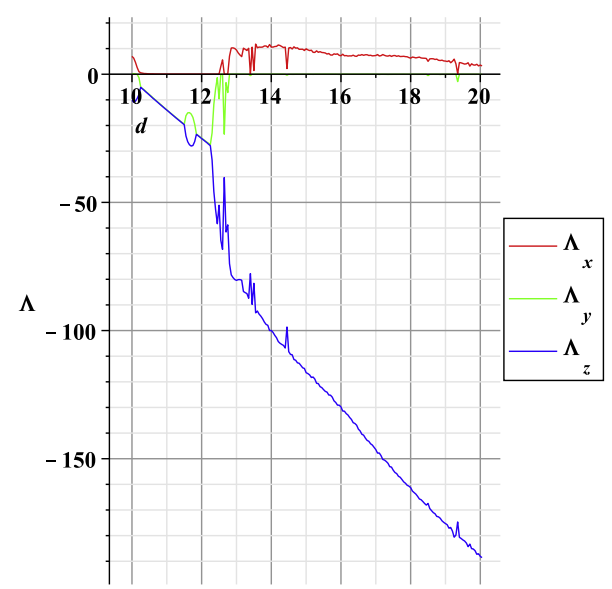

Fig. 22. Lyapunov exponents $\Lambda_{x}, \Lambda_{y}, \Lambda_{z}$ for system (23) at $c=10, d=10 \div 20$.

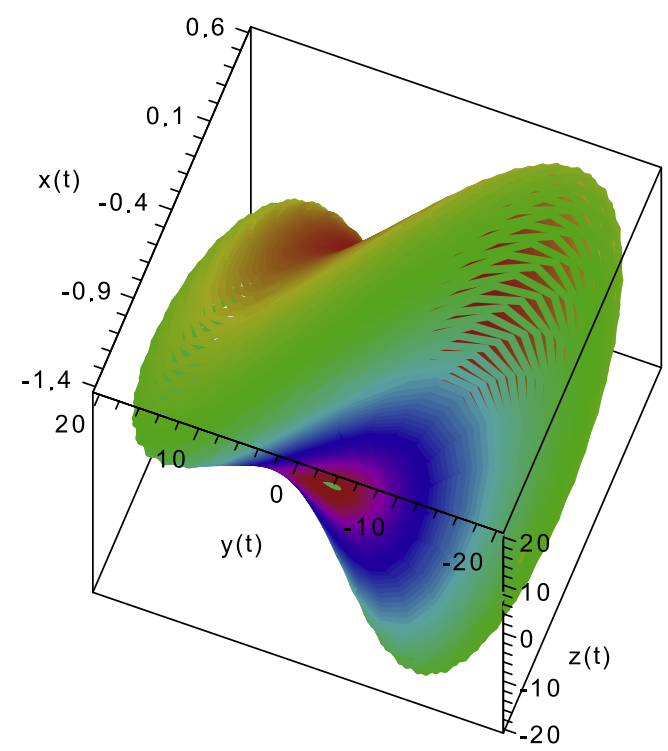

Fig. 23. Evolutions of the attractor of system (24) for $t=10$. 


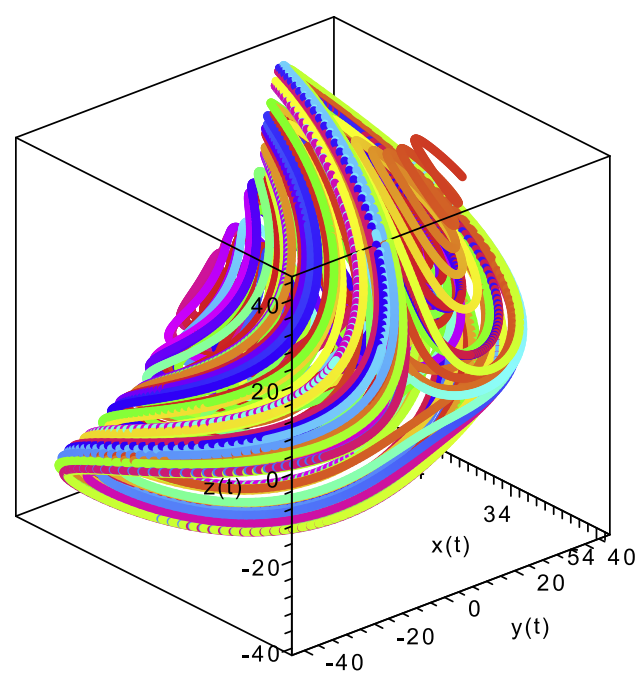

Fig. 24. Evolutions of the attractor of system (25) for $t=10$.

\section{Examples}

Below, on Figs. 12, 14, 15, 18, 20, 23, 24 for system (12) at different values of parameters, new types of chaotic attractors are represented. In addition on Figs. 13, 16, 17, 19, 21, 22 the Lyapunov exponents and Poincare mappings are shown. They verify the presence of the chaotic dynamics in the considered systems.

1. Consider the system depending on parameters $a$ and $b$ :

$$
\left\{\begin{array}{l}
\dot{x}(t)=3 x(t)-y^{2}(t)+z^{2}(t), \\
\dot{y}(t)=-1 y(t)-20 z(t)+a x(t) y(t), \\
\dot{z}(t)=x(t)+20 y(t)-1 z(t)-b x(t) z(t) .
\end{array}\right.
$$

2. Consider the system depending on parameters $c$ and $d$ :

$$
\left\{\begin{array}{l}
\dot{x}(t)=4 x(t)-y^{2}(t)+z^{2}(t), \\
\dot{y}(t)=-3 y(t)-250 z(t)+c x(t) y(t), \\
\dot{z}(t)=x(t)+250 y(t)-3 z(t)-d x(t) z(t) .
\end{array}\right.
$$

3. Consider the system

$$
\left\{\begin{array}{l}
\dot{x}(t)=-y^{2}(t)+z^{2}(t), \\
\dot{y}(t)=y(t)-250 z(t)+10 x(t) y(t), \\
\dot{z}(t)=250 y(t)+z(t)-15 x(t) z(t) .
\end{array}\right.
$$

4. Consider the system

$$
\left\{\begin{array}{l}
\dot{x}(t)=-5 x(t)+(1.6 y(t)+z(t))(y(t)-z(t)), \\
\dot{y}(t)=2 y(t)+28 z(t)-x(t) y(t) \\
\dot{z}(t)=x(t)-28 y(t)+2 z(t)+x(t) z(t) .
\end{array}\right.
$$

Finally, we notice that for systems (22), (23) the conditions of Theorem 5 (and its generalizations at $d_{1}=0, e_{1}=1$ ) are fulfilled. For systems (24), (25) these conditions dissatisfy.

\section{Conclusion}

This paper has reported the new 3-D smooth autonomous chaotic system (1) in which each equation has a quadratic term. New types of chaotic attractors can be generated from the system. (At some values of parameters in this system can exist Lorenz-like type attractors.) Basic properties of the new dynamical system have been analyzed by means of Lyapunov exponents and Poincare map. This analysis verifies the existence of chaotic dynamics in system (1). A detailed investigation of the characteristics of system (1) is left for the further studies. 


\section{Acknowledgments}

The author wishes to express his sincere gratitude to the anonymous referee for the helpful criticism and valuable suggestions which help to improve the paper.

\section{References}

[1] V.Ye. Belozyorov, On existence of homoclinic orbits for some types of autonomous quadratic systems differential equations, Applied Mathematics and Computation 217 (2011) 4582-4595.

[2] V.Ye. Belozyorov, New types of 3-D systems of quadratic differential equations with chaotic dynamics based on Ricker discrete population model, Applied Mathematics and Computation 218 (2011) 4546-4566.

[3] T. Zhou, G. Chen, Classification of chaos in 3-D autonomous quadratic systems - 1. Basic framework and methods, International Journal of Bifurifications Chaos 16 (9) (2006) 2459-2479.

[4] G. Qi, G. Chen G, M.A. van Wyk, B.J. van Wyk, Y. Zhang, A four-wing chaotic attractor generated from a new 3-D quadratic autonomous system, Chaos, Solitons and Fractals 38 (2008) 705-721.

[5] D. Shang, M. Han, The existence of homoclinic orbits to saddle-focus, Applied Mathematics and Computation 163 (2005) $621-631$.

[6] Z. Li, G. Chen, W.A. Halang, Homoclinic and heteroclinic orbits in a modified Lorenz system, Information Sciences 165 (2004) $235-245$.

[7] T. Zhou, G. Chen, Q. Yang, Constructing a new chaotic system based on the Shilnikov criterion, Chaos, Solitons and Fractals 19 (2004) 985-993.

[8] Y.A. Kuznetsov, Elements of Applied Bifurcation Theory, 2nd ed., Springer-Verlag, New-York, 1998.

[9] L. Wang, 3-scroll and 4-scroll chaotic attractors generated from a new 3-D quadratic autonomous system, Nonlinear Dynamics 56 (2009) $453-462$.

[10] B. Chen, T. Zhou, G. Chen, An extended Shilnikov homoclinic theorem and its applications, International Journal of Bifurifications Chaos 19 (5) (2009) 1679-1693.

[11] V. Ye. Belozyorov, Invariant approach to an existence problem of nontrivial asymptotic stability cone, Canadien Applied Mathematics Quarterly 15 (2) (2007) 125-168.

[12] X. Zhang, Y. Shi, G. Chen, Constructing chaotic polynomial maps, International Journal of Bifurifications Chaos 19 (2) (2009) $531-543$.

[13] R.M. Crownover, Introduction to Fractals and Chaos, Jones and Bartlett Publishers, Boston, London, 1995.

[14] A.C.J. Luo, Y. Guo, Parameter characteristics for stable and unstable solutions in nonlinear discrete dynamical systems, International Journal of Bifurifications Chaos 20 (10) (2010) 3173-3191.

[15] W. Liu, W.K.S. Tang, G. Chen, $2 \times 2$-scroll attractors generated in a three-dimensional smooth autonomous system, International Journal of Bifurifications Chaos 17 (11) (2007) 4153-4157.

[16] Q. Yang, G. Chen, A chaotic system with one saddle and two stable node-foci, International Journal of Bifurifications Chaos 18 (5) (2008) $1393-1414$.

[17] Y. Jiang, J. Sun, Shilnikov homoclinic orbits in a new chaotic system, Chaos, Solitons and Fractals 32 (2007) 150-159.

[18] J. Lu, G. Chen, D. Cheng, A new chaotic system and beyond: the generalized Lorenz-like system, International Journal of Bifurifications Chaos 14 (7) (2004) 1507-1537.

[19] Z. Chen, Y. Yang, Z. Yuan, A single three-wing or four-wing chaotic attractor generated from a three-dimensional smooth quadratic autonomous system, Chaos, Solitons and Fractals 38 (2008) 1187-1196.

[20] J. Wang, Z. Chen, Z. Yuan, Existence of a new three-dimensional chaotic attractors, Chaos, Solitons and Fractals 42 (2009) $3053-3057$.

[21] Q. Yang, Z. Wei, G. Chen, An unusual 3-D autonomous quadratic chaotic system with two stable node-foci, International Journal of Bifurifications Chaos 20 (4) (2010) 1061-1083.

[22] A.A. Yakubu, Two-patch dispersal-linked compensatory-overcompensatory spatially discrete population models, Journal of Biological Dynamics 1 (2) (2007) 157-182.

[23] W.E. Ricker, Stock and recruitment, Journal of Fisheries Research Board of Canada II (1954) 559-623. 